\title{
Structure and Rheology of Solutions and Gels of
}

\section{Stiff Polyelectrolyte at High Salt Concentration}

Olga E. Philippova, ${ }^{*}$ Andrey V. Shibaev, Dmitry A. Muravlev, and Dmitry Yu. Mityuk

Physics Department, Moscow State University, Moscow 119991, Russia, e-mail:

phil@polly.phys.msu.ru

\section{SUPPORTING INFORMATION}

A. Determination of the Content of Acetate and Pyruvate Groups. The content of acetate and pyruvate groups in xanthan was determined by ${ }^{1} \mathrm{H}-\mathrm{NMR}$ using Bruker AV-600 spectrometer. For this, $1 \mathrm{~g} / \mathrm{L}$ of xanthan (which corresponds to $1.06 \mathrm{mM}$ of monomer units) was dissolved in $\mathrm{D}_{2} \mathrm{O}$ with addition of $1 \mathrm{mM}$ sodium acetate as a quantification reference. The spectrum was recorded at $80^{\circ} \mathrm{C}$ and accumulated from 8 scans. A part of the spectrum corresponding to $-\mathrm{CH}_{3}$ groups is presented in Figure S1. According to the literature data [Roy, A.; Comesse, S.; Grisel, M.; Hucker, N.; Souguir, Z.; Renou F. Hydrophobically Modified Xanthan: An Amphiphilic but Not Associative Polymer. Biomacromolecules 2014, 15, 1160-1170], the peaks were attributed as follows: 2.04 ppm ( $-\mathrm{CH}_{3}$ of xanthan pyruvate groups), $2.47 \mathrm{ppm}$ (- $-\mathrm{CH}_{3}$ of sodium acetate), $2.73 \mathrm{ppm}\left(-\mathrm{CH}_{3}\right.$ of xanthan acetate groups). The average amounts of acetate and pyruvate groups (per one monomer unit) were found to be 0.56 and 0.41 , respectively.

\footnotetext{
* To whom correspondence should be addressed.
} 


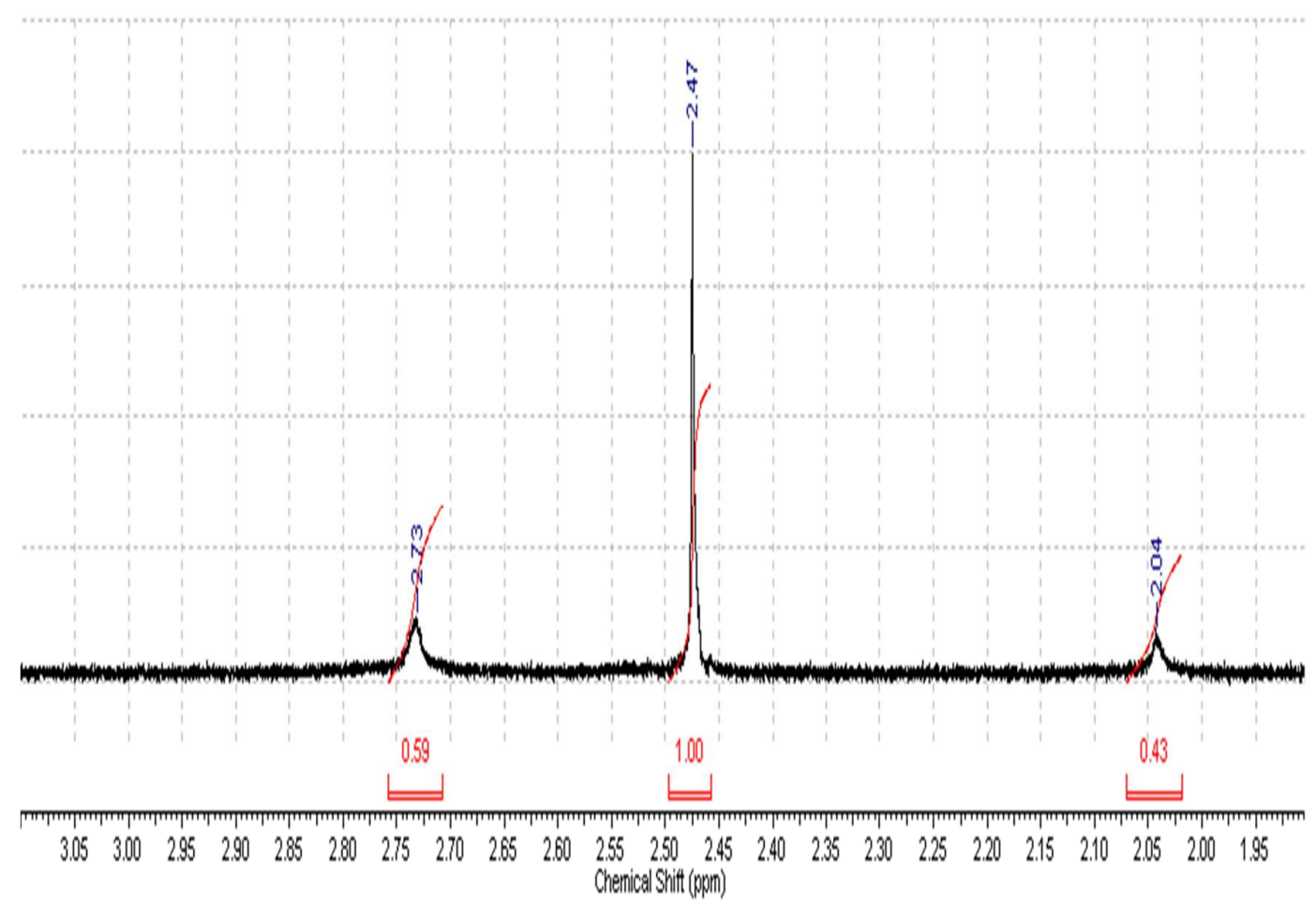

Figure S1. ${ }^{1} \mathrm{H}-\mathrm{NMR}$ spectrum of $1 \mathrm{~g} / \mathrm{L}$ xanthan and $1 \mathrm{mM}$ sodium acetate in $\mathrm{D}_{2} \mathrm{O}$ at $80^{\circ} \mathrm{C}$.

B. Construction of Rheological Master Curve. The master curves $b_{c} G^{\prime}\left(a_{c} \omega\right), b_{c} G^{\prime \prime}\left(a_{c} \omega\right)$ were obtained by horizontal and vertical shifts of the dynamic rheology data at different xanthan concentrations presented in Figure 7 of the main text. For this aim, the software IRIS RheoHub was used [Winter, H. H.; Mours, M. The Cyber Infrastructure Initiative for Rheology. Rheol. Acta 2006, $45,331-338]$. The reference concentration, relative to which all the data were shifted, is $1 \mathrm{wt} \%$. Horizontal $\left(a_{c}\right)$ and vertical $\left(b_{c}\right)$ shift factors for different xanthan concentrations are shown in Figure $\mathrm{S} 2$. It is seen that all the data except those for the lower polymer concentration $(0.09 \mathrm{wt} \%)$ coincide with the observed trend. Therefore, a single rheological master curve can be constructed by frequency-concentration superposition in the following range of xanthan concentrations: $0.15-1$ wt $\%$. 

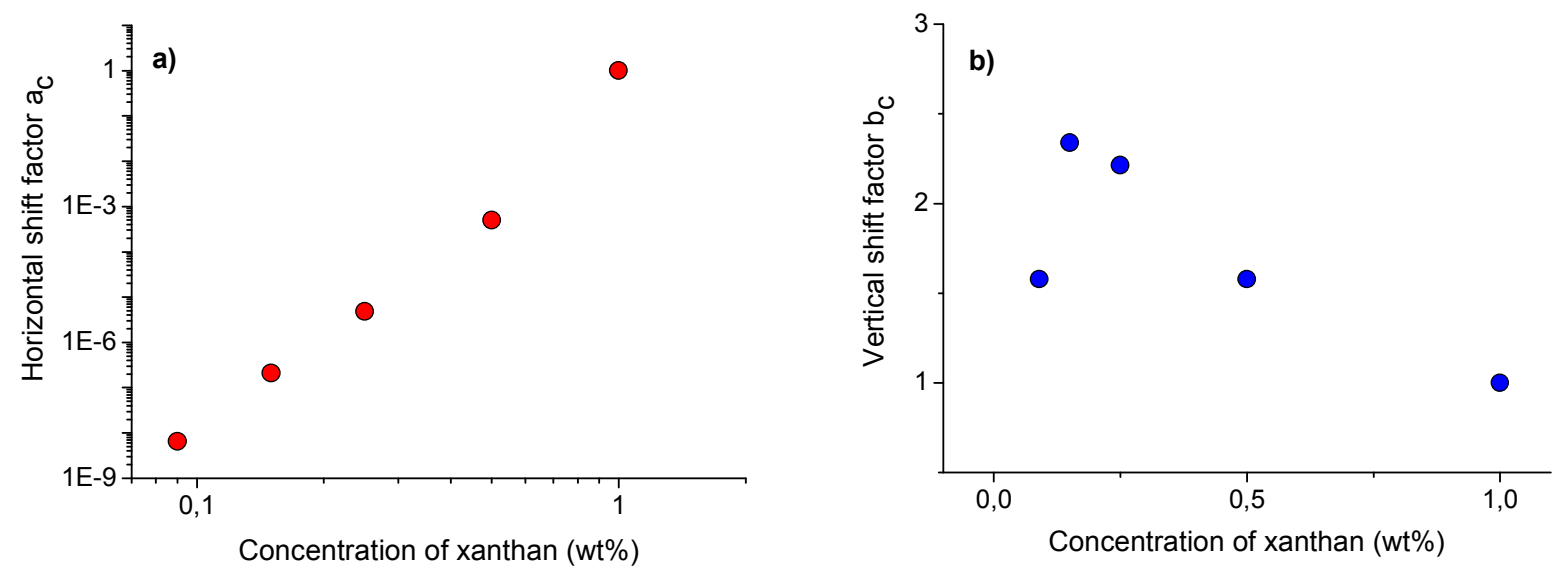

Figure S2. Horizontal (a) and vertical (b) shift factors of the frequency dependences of dynamic storage $\mathrm{G}^{\prime}$ and loss $\mathrm{G}^{\prime \prime}$ moduli of the aqueous solutions at different concentrations of xanthan $(0.09$ $1 \mathrm{wt} . \%$ ) in the presence of $4.75 \mathrm{wt} \% \mathrm{KCl}$.

C. SAXS of Xanthan Solutions and Gels. To get more information about the microstructure of xanthan solutions and gels SAXS was used. Typical scattering curve of xanthan solution in the absence of cross-linker is presented in Figure S3. The Guinier plot in the coordinates ln (Iq) vs. $\mathrm{q}^{2}$ (Figure S4) shows a linear slope, indicating the cylindrical structure. High-q region of this curve (at q values $0.03-0.15 \AA^{-1}$ ) relevant to smaller objects is well fitted by a cylinder model with radius of $13 \AA$, which perfectly corresponds to the radius of double helix (12 $\AA$ [Viebke, C. Order-Disorder Conformational Transition of Xanthan Gum. In Polysaccharides: Structural Diversity and Functional Versatility. Ed. by Dumitriu, S. Boca Raton: CRC Press, $2^{\text {nd }}$ ed., 2004, pp. 459-474]) formed by xanthan macromolecules. Similar value of the radius (12.9 $\AA)$ was obtained from the slope of the Guinier plot $\mathrm{R}^{2} / 2=83.3 \AA^{2}$ (Figure S4). According to FF-TEM data, one can also expect the structural heterogeneities on larger length scales, but these are outside the q-range accessible in SAXS. 


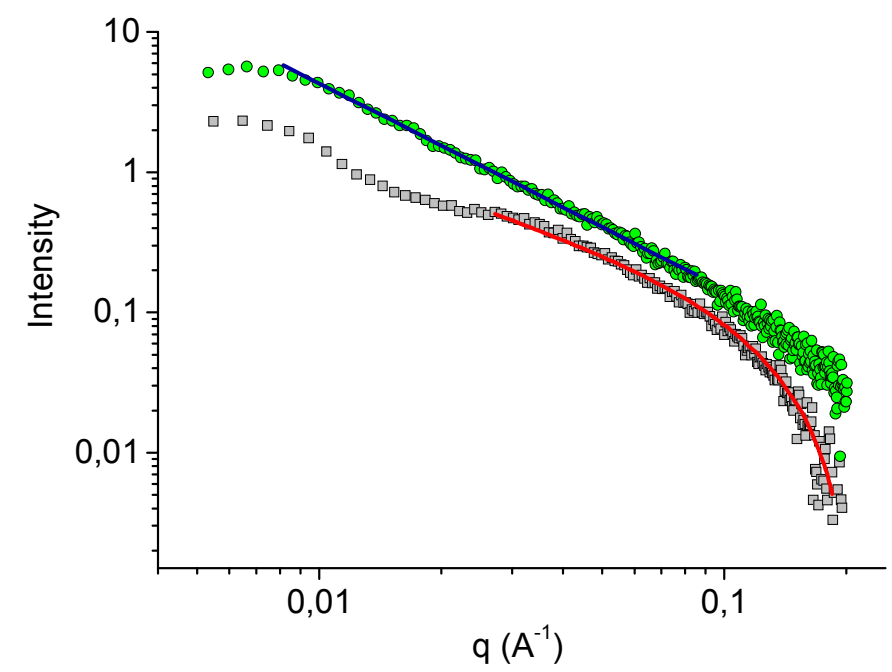

Figure S3. Scattering curves for $1.05 \mathrm{wt} \%$ solutions of xanthan before (squares) and after crosslinking by chromium (circles) (molar ratio of chromium ions to xanthan monomer units - 0.2). Solid lines represent the fits by a cylinder (red line) or a fractal structure with $\mathrm{I} \sim \mathrm{q}^{-\mu}$ (blue line). Solvent: $4.75 \mathrm{wt} \% \mathrm{KCl}$ in $\mathrm{D}_{2} \mathrm{O}$.

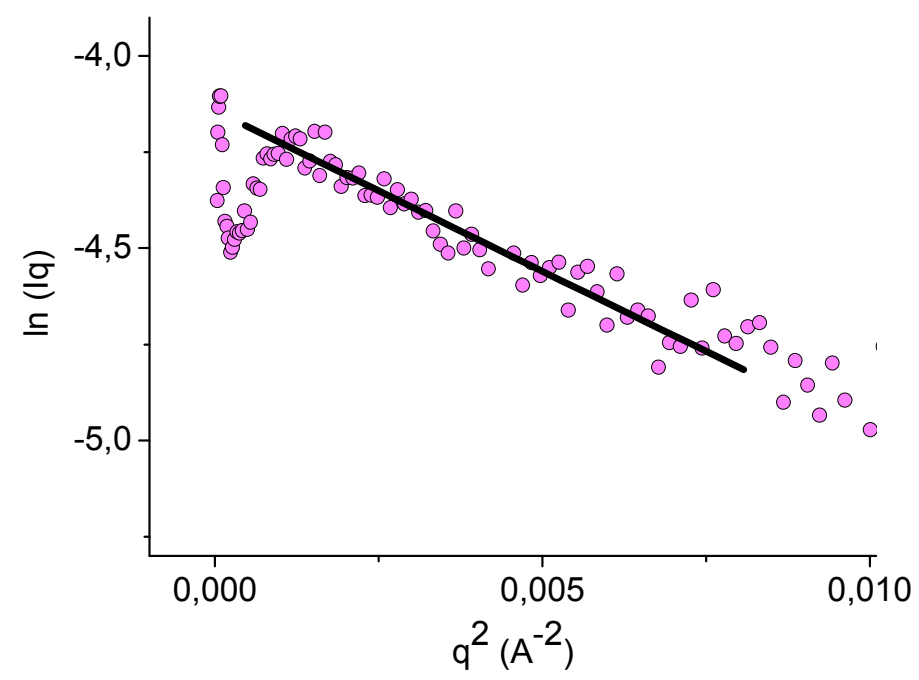

Figure S4. Guinier plot for SAXS data taken from $1.05 \mathrm{wt} \%$ solution of xanthan in $4.75 \mathrm{wt} \% \mathrm{KCl}$ in $\mathrm{D}_{2} \mathrm{O}$. Solid line has a slope of $83.3 \AA^{2}$. 
The scattering curve for xanthan gel after cross-linking by chromium (III) is presented in Figure S3. The double-logarithmic plot (Figure S3) shows a presence of wide linear region spanning the qrange from 0.01 to $0.07 \AA^{-1}$ giving a fractal dimension of 1.45 .

D. Histograms Obtained from FF-TEM Data. From FF-TEM data the distribution of lengths of rod-like fragments of ordered xanthan macromolecules was determined. The results are presented in Figure S5. It is seen that the average length of these fragments $(76 \mathrm{~nm})$ is smaller than the persistence length $(120 \mathrm{~nm})$ [Sato, T.; Norisuye, T.; Fujita, H. Double-Stranded Helix of Xanthan: Dimensional and Hydrodynamic Properties in 0.1M Aqueous Sodium Chloride. Macromolecules 1984, 17, 2696-2700].

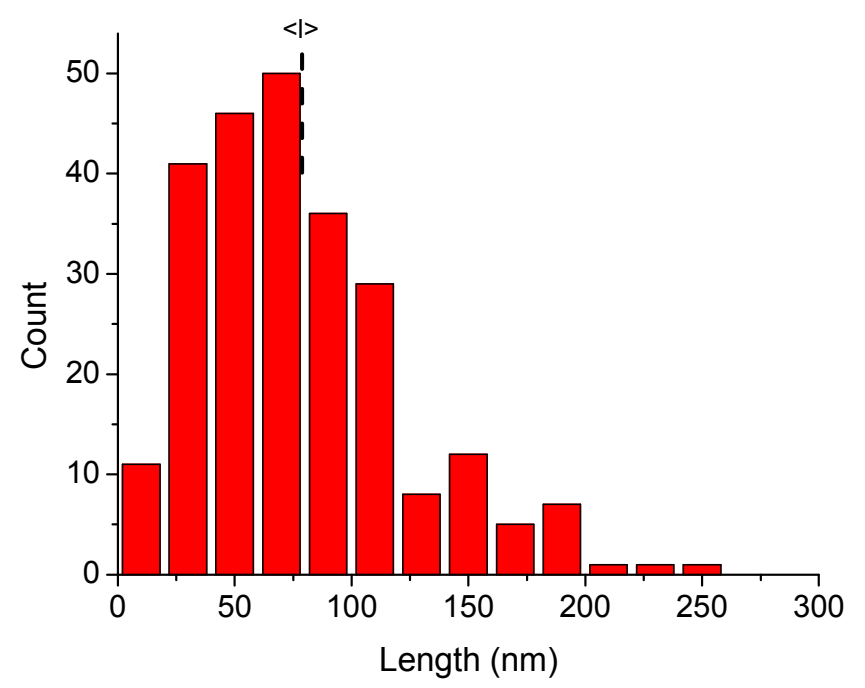

Figure S5. Histogram of distribution of the lengths of rod-like fragments in $0.15 \mathrm{wt} \%$ aqueous solution of xanthan in the presence of $4.75 \mathrm{wt} \% \mathrm{KCl}$ obtained from FF-TEM data.

To characterize the aggregation of macromolecules composing the skeleton of the network the histograms of the distribution of the thickness of skeleton were obtained from FF-TEM data (Figure 
S6). They show that the aggregation increases with increasing polymer concentration and at the cross-linking of xanthan chains by chromium.
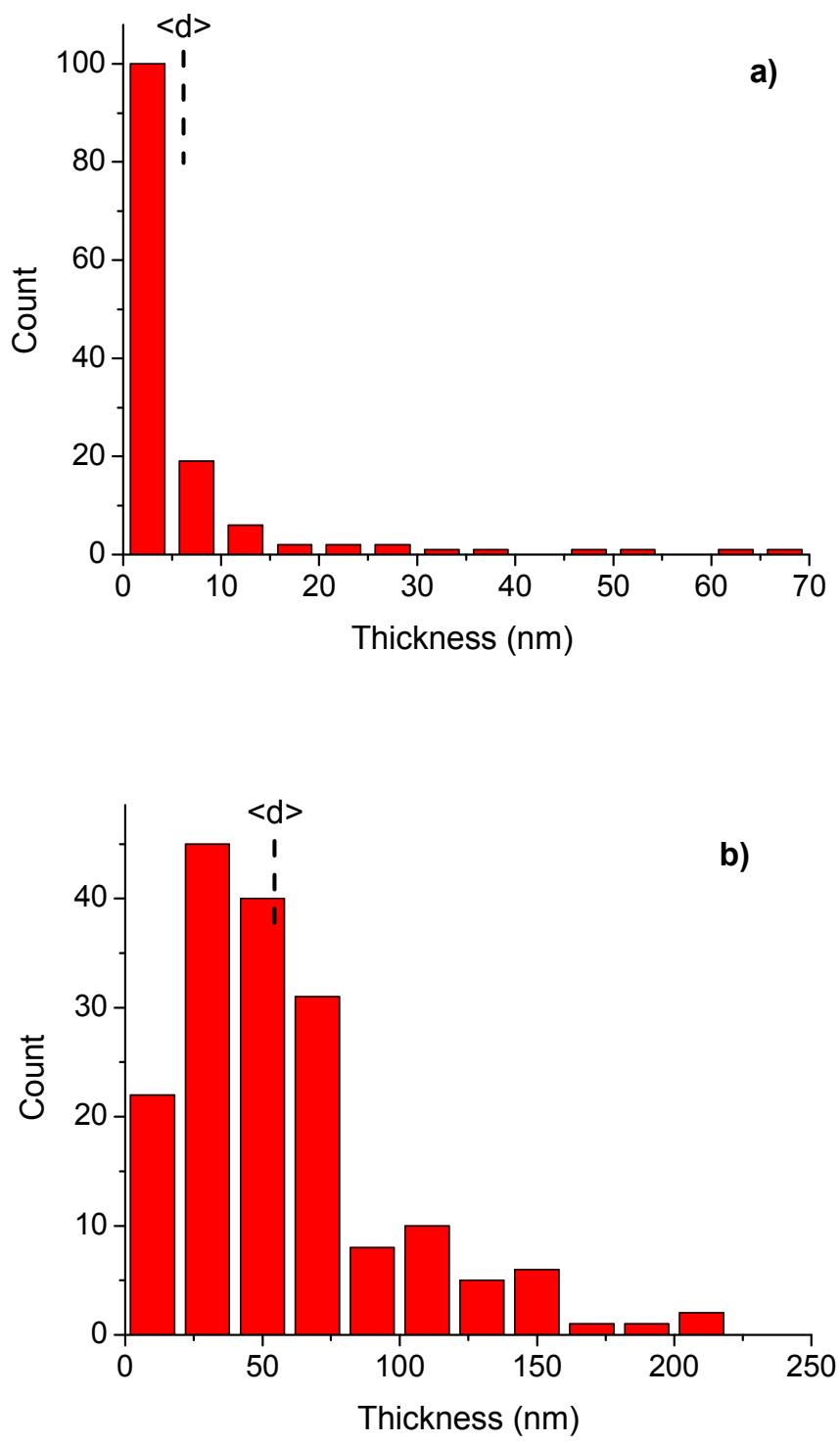

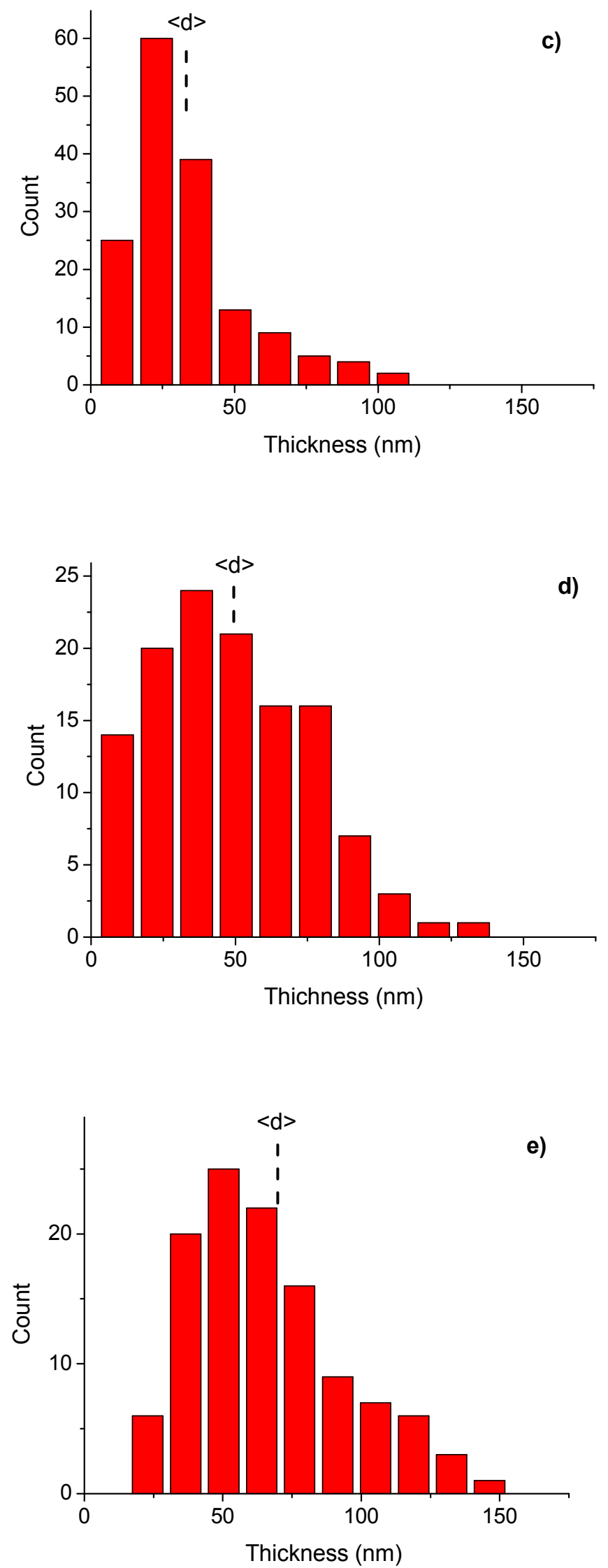
Figure S6. Histograms of distribution of thickness of polymer-rich skeleton in 0.15 (a) and 0.5 wt $\%$ (b) xanthan solutions and in 0.03 (c), 0.09 (d) and $0.15 \mathrm{wt} \%$ (e) xanthan gels cross-linked by chromium chloride. Solvent: $4.75 \mathrm{wt} \% \mathrm{KCl}$ in water; molar ratio of chromium ions to xanthan monomer units is 0.2 . 